\title{
A INFLUENNCIA DA ORIGEM DAS EMPRESAS NO GERENCIAMENTO E NA AVALIAÇÃO DE DESEMPENHO ORGANIZACIONAL
}

\author{
THE INFLUENCE OF ORIGIN OF COMPANIES \\ IN MANAGEMENT AND ORGANIZATIONAL \\ PERFORMANCE ASSESSMENT
}

\author{
Recebido: 08-10-2014 \\ Aceito: 24-02-2015 \\ Leonardo Fabris Lugoboni ${ }^{1}$ \\ Marcus Vinicius Moreira Zittei ${ }^{2}$ \\ Roger Marques Lopes ${ }^{3}$ \\ Joice Chiareto ${ }^{4}$ \\ Hamilton Luiz Correa ${ }^{5}$ \\ Maria Laura Ferranty Mac Lennan ${ }^{6}$
}

\section{RESUMO}

Este trabalho estudou a influência da origem cultural de três empresas multinacionais no gerencimento e na avaliação do desempenho organizacional. As organizações estudadas possuem origem alemã, francesa e finlandesa com expressão no mercado de energia. Foram realizadas, com base no modelo das quatro dimensões culturais de Hofstede (2010) e na literatura de avaliação de desempenho organizacional, entrevistas com gestores dessas organizações. Os resultados indicam que a origem cultural possui influência na gestão do desempenho. Porém, foram encontrados, também, alguns resultados contraditórios ao modelo de Hofstede, o que poderia ser explicado pela flexibilização das fronteiras culturais gerada pela globalização.

Palavras-chave: Gestão de desempenho, Dimensões da cultura, Hofstede, Cultura Organizacional.

1 Possui graduação em Administração pelo Centro Universitário Fundação Santo André - CUFSA. Mestrado em Administração pela Universidade Municipal de São Caetano do Sul - USCS. Atualmente é doutorando em Administração pela Universidade de São Paulo - USP. São Paulo, São Paulo. Brasil. E-mail: leo_fabris@hotmail.com

2 Possui graduação em Ciências Contábeis pelo Centro Universitário FIEO - UNIFIEO. Mestrado em Ciências Contábeis pelo Centro Universitário FECAP. Atualmente é doutorando em Ciências Contábeis pela Fundação Universidade Regional de Blumenau FURB. São Paulo, São Paulo. Brasil. E-mail: marcuszittei@zittei.com.br

3 Atualmente é estudante no Centro Universitário FECAP. São Paulo, São Paulo. Brasil. E-mail: rogermlopes@gmail.com

4 Possui graduação em Administração pela Universidade Paranaense - UNIPAR. Mestrado em Administração pela Universidade de São Paulo - USP. Atualmente é doutoranda em Administração na Universidade de São Paulo - USP. São Paulo, São Paulo. Brasil. E-mail: joice.chiareto@gmail.com

5 Possui graduação, mestrado e doutorado em Administração em pela Universidade de São Paulo - USP. Atualmente é professora na Universidade de São Paulo. São Paulo, São Paulo. Brasil. E-mail: hamilco@usp.br

6 Possui graduação em Adminsitração pela Universidade de São Paulo - USP. Mestrado em Administração pela Escola Superior de Propanda e Marketing - ESPM. Atualmente é doutorando em Administração pela Universidade de São Paulo - USP. São Paulo, São Paulo. Brasil. E-mail: ferranty@hotmail.com 


\section{ABSTRACT}

This work studied the influence of the cultural origin of three companies multinationals in the management and evaluation of the organizational performance. The organizations studied have German, French and Finnish origins with expression in the energy market. It was performed interviews, with managers of these organizations, which were based on the model of the four cultural dimensions of Hofstede (2010) and the evaluation of organizational performance literature. The results indicate that origin of culture has influence on performance management, but have also found some contradictory results to model of Hofstede, which could be explained by relaxing of cultural boundaries generated by globalization results.

Keywords: Performance Management, Dimensions of culture, Hofstede, Organizational Culture.

\section{INTRODUÇÃO}

O mercado tem se mostrado cada vez mais competitivo em qualquer região ou área de atuação, tornando a avaliação da administração das organizações, devido à dinâmica que as envolve, uma tarefa extremamente complexa (DRUCKER, 1964). Kaplan e Norton (1997, p. 21) reforçam a importância e a inerente dificuldade da mensuração e avaliação do desempenho de uma organização, afirmando que "medir é importante: o que não é medido não é gerenciado".

É preocupação recorrente de administradores de diversos tipos de organizações e setores conhecer o desempenho das organizações que dirigem. Desde o final do século XIX até os dias de hoje, foi concebida uma significativa quantidade de sistemas de medição de desempenho organizacional, cada um deles visando atender a demandas específicas (SOBREIRA NETTO, 2007).

Entretanto, um dos grandes desafios enfrentados pelos administradores para gerenciamento de desempenho é o fenômeno da globalização, que afeta todas as organizações (MOREIRA, 2009). Pode-se entender como globalização a situação em que bens, serviços, pessoas, habilidades e ideias cruzam livremente as fronteiras geográficas (LUGOBONI, 2010); trata-se de um processo de interação acelerado e complexo de integração e crescimento da interdependência em diversas regiões do mundo (PRASAD, 2007).

Ao tratar de globalização, para Ardalan (2008), as prioridades são: crescimento econômico, livre comércio para estimular o crescimento, mercado sem restrições, escolhas independentes, redução de regulamentações governamentais; e defesa de um modelo evolutivo de desenvolvimento social, baseado na experiência ocidental e aplicável ao resto do mundo.

Nesse contexto, observa-se que o fator cultural desempenha um papel fundamental no processo de globalização, o que, consequentemente, influencia o gerenciamento do desempenho pelos administradores. Swanson George (1974) e Perrim (1998), diante disso, ressaltam a importância do contexto cultural no surgimento das teorias administrativas de controle de desempenho e a necessidade de adequação de tais perspectivas à aplicação dos sistemas de monitoramento do desempenho na tomada de decisões. Neely (1999) ressalta, entretanto, que a pertinência e validade das medidas de desempeho podem variar de acordo com o país e o cenário cultural ao qual pertence.

Em termos de desenvolvimento econômico, o mercado de energia destaca-se por ser considerado um setor essencial e com uma das maiores tendências de crescimento para o futuro (THE FUTURIST, 2009). As organizações desse setor são extremamente internacionalizadas e afetadas pela globalização, motivo pelo qual estão sujeitas a sofrerem grandes impactos da cultura.

Tendo isso em vista, o objetivo geral desta pesquisa é compreender como os aspectos culturais influenciam a forma que as empresas do ramo de energia de diferentes origens gerenciam e avaliam seu desempenho. Apesar de se voltar a um campo específico, esta pesquisa é importante aos administradores de empresas de qualquer ramo para o entendimento da influência cultural em suas organizações, auxiliando na definição de seus métodos de avaliação de desempenho. 


\title{
2 FUNDAMENTAÇÃO TEÓRICA
}

\subsection{Cultura}

Linton (1945) afirma que a cultura é o modo de vida de uma sociedade, incluindo muitos detalhes de conduta, mas todos com certos fatores em comum, como crenças, costumes e valores. Esses fatores representam as reações normais e antecipadas de qualquer membro da sociedade a uma situação em particular.

Hofstede (2010) relata que a cultura é uma programação mental, ou seja, o "software da mente", produzido no ambiente social em que a pessoa cresce e adquire suas experiências. Ele considera que essa programação coletiva da mente é o que distingue os membros de um grupo ou de uma categoria de pessoas de outros. Ainda segundo Hofstede, a cultura é um fenômeno em si mesmo, residindo no fato de que as culturas nacionais têm como elemento central os valores, os quais são adquiridos na família, na comunidade e na escola.

\subsection{Cultura organizacional}

De forma menos abrangente, a cultura das organizações também pode apresentar distinções entre si. Foguel (2010) cita três conceitos de autores que definem a cultura organizacional como:

\begin{abstract}
O conjunto de pressupostos básicos, de valores, inventados, descobertos ou desenvolvidos por um grupo para resolver problemas tanto na adaptação externa e/ou integração interna e que funcionaram bem o suficiente para serem considerados válidos e ensinados a novos membros como forma correta de perceber, pensar e sentir, em relação a estes problemas (SCHEIN, 1986, p. 38).
\end{abstract}

Valores e crenças compartilhados pelos membros de uma organização, a qual funciona como um mecanismo de controle organizacional, informalmente aprovando ou proibindo comportamentos e que dá significado, direção e mobilização para os membros da organização (SANTOS, 2000, p. 29).

Um poderoso mecanismo de controle, que visa conformar condutas, homogeneizar maneiras de pensar e viver a organização (FREITAS, 1991, p. 76).

Pode-se afirmar, então, que a cultura organizacional é constituída por todas as características de uma organização que definem a sua "personalidade" e que permitem, em seu conjunto, distingui-la das outras. Assim, toda organização, por mais simples que seja, possui uma cultura própria em que se manifestam seus valores, suas crenças, seus costumes, suas tradições e seus símbolos, podento também estar relacionada com o modo como as organizações encaram seus problemas (SANTOS, 2011).

Um fator que possui grande relação com a cultura organizacional é o modo como as decisões são tomadas. Rowe e Boulgarides (1983 apud PODRUG, 2006) sugerem que o estilo de tomada de decisão é uma forma útil para que se entendam os estilos de gerenciamento. Via de regra, a classificação dos estilos de tomada de decisão varia entre autocracia e democracia, com diversas diferenças. A seguir, no Quadro 1, apresenta-se uma tipologia de estilos de tomada de decisão utilizada para pesquisas empíricas.

\begin{tabular}{|c|l|}
\hline Tipo & \multicolumn{1}{c|}{ Definição de estilo de decisão } \\
\hline Autocrático & $\begin{array}{l}\text { Na maioria das vezes, resolve o problema ou toma decisão por meio de } \\
\text { informações disponíveis sem consultar o(s) subordinado(s). }\end{array}$ \\
\hline
\end{tabular}




\begin{tabular}{|c|l|}
\hline Pseudo consultivo & $\begin{array}{l}\text { Na maioria das vezes, consulta o(s) subordinado(s), mas isso não significa } \\
\text { que considera suas ideias e sugestões. }\end{array}$ \\
\hline Consultivo & $\begin{array}{l}\text { Na maioria das vezes, consulta previamente o(s) subordinado(s), tomando } \\
\text { decisões que podem ou não refletir a influência dos subordinados. }\end{array}$ \\
\hline Participativo & $\begin{array}{l}\text { Na maioria das vezes, compartilha e analisa problemas com os subordina- } \\
\text { dos como um grupo, avalia alternativas e chega a uma decisão da maioria. }\end{array}$ \\
\hline Pseudo participativo & $\begin{array}{l}\text { Na maioria das vezes, compartilha e analisa os problemas com os subordi- } \\
\text { nados como um grupo e avalia alternativas para determinar a decisão certa, } \\
\text { mas os informa com antecedência sobre o que acha que é o caminho certo } \\
\text { e depois toma a decisão por meio do voto. }\end{array}$ \\
\hline Delegatório & Na maioria das vezes, pede aos subordinados para tomar decisões por si mesmos. \\
\hline
\end{tabular}

Quadro 1 - Definições dos tipos de tomada de decisão

Fonte: Ali (1993 apud PODRUG, 2006).

Para Podrug (2006) a tomada de decisão é culturalmente contingente, o que significa que aquilo entendido como a "melhor prática" depende de valores, crenças, atitudes e padrões de comportamento das pessoas envolvidas.

\subsubsection{Dimensões de Hofstede}

Geert Hofstede pesquisou entre os anos 60 e 70, nos escritórios da IBM de todo o mundo, as atitudes dos empregados, utilizando um único tipo de questionário. Seu estudo abarcou as diferenças culturais existentes em vários países a fim de verificar a importância da cultura nacional na forma de administrar. Em seu estudo, realizado em 76 países e três regiões continentais (África Ocidental, África Oriental e países de língua árabe), resultado de mais de 116 mil questionários, o autor encontrou diferenças significativas entre comportamentos e atitudes dos empregados.

Ele pôde constatar a influência da cultura nacional para explicar valores e atitudes em relação ao trabalho e, a partir desse estudo, desenvolveu o primeiro modelo empírico de "dimensões" da cultura nacional: As Dimensões de Hofstede (HOFSTEDE, 2010).

A base do trabalho de Hofstede é agrupar administradores e funcionários ao longo de quatro dimensões de cultura: Distância do Poder, Coletivismo versus Individualismo, Feminilidade versus Masculinidade e Controle da Incerteza (CASAGRANDE, 2009). No Quadro 2, estão resumidos os principais conceitos das dimensões culturais de acordo com o estudo de Hofstede.

\begin{tabular}{|c|l|}
\hline Dimensão & \multicolumn{1}{c|}{ Conceito desenvolvido por Hofstede } \\
\hline Distância do Poder & $\begin{array}{l}\text { A distância do poder é a medida de quanto os membros menos poderosos de uma } \\
\text { civilização aceitam e esperam distribuição desigual de poder na sociedade. Nos países } \\
\text { onde há um indicador baixo, a dependência dos funcionários quanto às chefias é me- } \\
\text { nor. Quando a distância é grande, superiores e subordinados consideram-se desiguais } \\
\text { por natureza e existe maior dependência dos empregados em relação às chefias. }\end{array}$ \\
\hline $\begin{array}{c}\text { Individualismo versus } \\
\text { Coletivismo }\end{array}$ & $\begin{array}{l}\text { Nas sociedades coletivistas, prevalece o interesse do grupo sobre o interesse individu- } \\
\text { al. Nas individualistas, por sua vez, prevalece o interesse do indivíduo sobre o grupo. } \\
\text { Essa dimensão se associa com a importância atribuída ao tempo pessoal, à liberdade, } \\
\text { ao desafio, à formação, às condições de trabalho e à utilização de competências. }\end{array}$ \\
\hline $\begin{array}{c}\text { Feminilidade versus } \\
\text { Masculinidade }\end{array}$ & $\begin{array}{l}\text { A feminilidade é uma medida do quanto tarefas e características consideradas } \\
\text { femininas são compartilhadas pelos homens em uma sociedade. Características } \\
\text { masculinas estão ligadas à oferta de mais oportunidades de reconhecimento, pro- } \\
\text { moção e possibilidades de enfrentar novos desafios; já as femininas referem-se ao } \\
\text { oferecimento de mais oportunidades de ajuda mútua e de contatos sociais. }\end{array}$ \\
\hline Controle da Incerteza & $\begin{array}{c}\text { Essa dimensão identifica como as sociedades se comportam em relação ao } \\
\text { desconhecido, que gera incerteza e riscos. }\end{array}$ \\
\hline
\end{tabular}

Quadro 2 - Estilos de tomada de decisão

Fonte: Hofstede (2010) 
Apesar da grande aceitação, o trabalho de Hofstede também recebeu críticas, tais como: igualar nação com cultura, dificuldades e limitações de quantificar a cultura e o status do observador fora da cultura, e confiança de que existe estabilidade nas diferenças culturais (BASKERVILLE, 2003).

\subsection{Avaliação de desempenho}

A avaliação de desempenho organizacional é um conceito abrangente e apresenta grande complexidade, principalmente devido às diversas metodologias e abordagens existentes para avaliar o desempenho. A avaliação de desempenho tem dois focos: avaliação de desempenho para fins internos (gerencial) e avaliação de desempenho para fins externos (BORTOLUZZI, 2011). Cabe salientar que este trabalho tem como foco a avaliação de desempenho para fins internos (gerencial).

A avaliação de desempenho deve atender a alguns requisitos: (i) o que vai ser avaliado ou seja, conhecer o objeto da avaliação, incluindo a sua identidade, a cultura sobre a qual essa identidade é construída e as instâncias que respondem pelo objeto a ser avaliado; (ii) como proceder à avaliação - ou seja, identificar como cada objetivo será avaliado e o quanto cada objetivo contribui para a avaliação do todo; e (iii) como conduzir ao gerenciamento interno - com base na análise das fragilidades e potencialidades identificadas para sugerir ações de aperfeiçoamento -, promovendo a alavancagem do desempenho institucional (IGARASHI, 2008 apud BORTOLUZZI, 2011).

Os sistemas de medição de desempenho acompanharam a evolução dos modelos organizacionais em seus conceitos básicos. Uma pesquisa realizada pela KPMG LLP (2001), resumida no Quadro 3, avalia a forma como a mensuração de desempenho se alterou durante o passar dos anos, diminuindo o foco na dimensão financeira (multidimensionalidade) e acrescentando outros conceitos como, por exemplo, as relações causa-efeito (Balanced Scorecard), a avaliação do capital intelectual (Skandia) e avaliação da sustentabilidade (Sigma).

Após consultar os autores Andriessen, Deprez e Tissen (2000); Azevedo e Miranda (2000); Bourne; Kennerley e Franco-Santos, 2005; Corrêa (1986); Corrêa, Hourneaux e Ruiz (2005); Costa (2001); Fagundes e Feliu (2007); Kaplan e Norton (1992, 1993, 1996, 1997, 2000a, 2000b, 2001, 2004a, 2004b, 2006); Kennerley e Neely (2002a e 2000b); KPMG (2001), foi possível agrupar os principais modelos de avaliação de desempenho e suas características no Quadro 3, exposto a seguir.

\begin{tabular}{|c|c|c|c|}
\hline $\begin{array}{c}\text { NOME DO } \\
\text { MODELO }\end{array}$ & $\begin{array}{c}\text { LíDERES } \\
\text { PRESUMIDOS } \\
\text { e DATA DE } \\
\text { ORIGEM }\end{array}$ & IDEIA PRINCIPAL e PRICIPAIS CARACTERÍSTICAS \\
\hline $\begin{array}{c}\text { Painel de } \\
\text { controle } \\
\text { de bordo } \\
\text { (Tableau } \\
\text { de Bord) }\end{array}$ & $\begin{array}{c}\text { Engenheiros } \\
\text { de processos } \\
\text { franceses } \\
\text { 1930s-1940s }\end{array}$ & $\begin{array}{l}\text { Painel para identificar relações de causa e efeito (antecessor ao conceito do BSC). } \\
\text { Promove a cada gerente uma visão geral e concisa do desempenho de sua unida- } \\
\text { de para guiar a tomada de decisão; informa o próximo nível sobre o desempenho } \\
\text { de cada unidade; força cada unidade a posicionar-se com relação ao contexto da } \\
\text { estratégia global da empresa e com relação às responsabilidades das demais uni- } \\
\text { dades; identifica os fatores críticos de sucesso e os indicadores-chave de desem- } \\
\text { penho; contribui para estruturar a agenda e dirigir o foco e as discussões. }\end{array}$ \\
\hline $\begin{array}{c}\text { Método das } \\
\text { Áreas-Chave } \\
\text { de Resultado }\end{array}$ & $\begin{array}{c}\text { R. Cordiner } \\
\text { (CEO-GE) } \\
1955\end{array}$ & $\begin{array}{l}\text { Usa oito pontos-chave: lucratividade; posição de mercado; produtividade; lideran- } \\
\text { ca de produto; desenvolvimento de pessoal; atitude dos empregados; responsa- } \\
\text { bilidade pública; e equilíbrio, em objetivos de curto e longo prazo. Pode ser adap- } \\
\text { tado e implementado em outras empresas, sem afetar a eficácia do modelo. A } \\
\text { mensuração deve atender aos objetivos de curto e de longo prazo e deve ocorrer } \\
\text { minimamente em todos os níveis da estrutura organizacional. }\end{array}$ \\
\hline $\begin{array}{c}\text { Método de Bu- } \\
\text { chele }\end{array}$ & $\begin{array}{c}\text { Robert } \\
\text { Buchele } \\
1970\end{array}$ & $\begin{array}{l}\text { Possui foco na análise da força competitiva dos principais departamentos, sobre- } \\
\text { tudo da área financeira e da administração da cúpula. Focaliza claramente índices } \\
\text { vitais para a organização; é facilmente aplicável; ajuda o avaliador a se certificar } \\
\text { de que não esqueceu nada; e verifica o desempenho em cada um dos processos. }\end{array}$ \\
\hline
\end{tabular}




\begin{tabular}{|c|c|c|}
\hline $\begin{array}{l}\text { NOME DO } \\
\text { MODELO }\end{array}$ & \begin{tabular}{|l|} 
LÍDERES \\
PRESUMIDOS \\
e DATA DE \\
ORIGEM \\
\end{tabular} & IDEIA PRINCIPAL e PRICIPAIS CARACTERÍSTICAS \\
\hline \begin{tabular}{|c|} 
O Método da \\
Avaliação Global \\
de Desempenho \\
de Corrêa-MA- \\
DE-O \\
\end{tabular} & $\begin{array}{l}\text { H. L. Corrêa } \\
1986\end{array}$ & $\begin{array}{l}\text { Verifica as causas de desempenho dentro de cada módulo de desempenho da organi- } \\
\text { zação. Usa mecanismos de análise crítica do desempenho global; estimula aprendizado; } \\
\text { usa múltiplas dimensões de desempenho; identifica relações de causa e efeito; busca o } \\
\text { alinhamento com a estratégia e a estrutura organizacional por meio da adaptação dos } \\
\text { módulos e indicadores; e usa métodos de avaliação qualitativos e quantitativos. }\end{array}$ \\
\hline $\begin{array}{c}\text { BSC - } \\
\text { Balanced } \\
\text { Scorecard }\end{array}$ & $\begin{array}{l}\text { Robert S. } \\
\text { Kaplan e Da- } \\
\text { vid P. Norton } \\
\text { 1990s }\end{array}$ & $\begin{array}{l}\text { Scorecard para medir e estabelecer relações de causa e efeito de quatro perspectivas } \\
\text { sobre processos: financeiros, de clientes, internos e crescimento. Apresenta lógica } \\
\text { convincente; correlação clara entre indicadores e desempenho financeiro; relações } \\
\text { de causa e efeito; possibilidade de adaptação para um sistema de gerenciamento de } \\
\text { patrimônio intelectual; e literatura bem desenvolvida e consistente, sendo bastante } \\
\text { utilizado, na prática, para formular estratégia e ganhar comprometimento interno. }\end{array}$ \\
\hline \begin{tabular}{l|} 
PMQ - \\
Performance \\
Measure Ques- \\
tionnaire
\end{tabular} & $\begin{array}{c}\text { Dixon, Nanni e } \\
\text { Vollmann } \\
1990\end{array}$ & $\begin{array}{l}\text { Avalia a efetividade do sistema de medição de desempenho e identifica as necessidades } \\
\text { de melhoria das organizações. Mecanismos de análise crítica do desempenho global faci- } \\
\text { litam a avaliação organizacional, estimulam a participação dos funcionários no processo } \\
\text { de avaliação do modelo de avaliação e facilitam o aprendizado organizacional. }\end{array}$ \\
\hline $\begin{array}{l}\text { SMART - Stra- } \\
\text { tegic Measu- } \\
\text { rement and } \\
\text { Reporting Tech- } \\
\text { nique }\end{array}$ & $\begin{array}{c}\text { Lynch e Cross } \\
1991\end{array}$ & $\begin{array}{l}\text { Leva a visão da organização, traduzida em objetivos financeiros, aos sistemas de ope- } \\
\text { ração do negócio, de forma que as medidas operacionais sustentem a visão da organi- } \\
\text { zação. Usa medidas vinculadas à estratégia, desdobrando as medidas de desempenho } \\
\text { do nível estratégico ao operacional, bem como múltiplas dimensões de desempenho, } \\
\text { e apresenta relações de causa e efeito entre os indicadores e os objetivos. }\end{array}$ \\
\hline $\begin{array}{c}\text { SCD - Sete Crité- } \\
\text { rios de Desem- } \\
\text { penho }\end{array}$ & $\begin{array}{c}\text { Sink e Tuttle } \\
1993\end{array}$ & $\begin{array}{l}\text { Avalia o desempenho global da organização por meio de todos os seus pontos- } \\
\text { chave. Usa medidas de desempenho em todos os pontos-chave (sistemas recep- } \\
\text { tores, saídas, processos, entradas e sistemas fornecedores) e apresenta múltiplas } \\
\text { dimensões de desempenho e mecanismos de realimentação que fornecem infor- } \\
\text { mações do resultado das ações de melhoria contínua. }\end{array}$ \\
\hline $\begin{array}{l}\text { MQMD - Mo- } \\
\text { delo Quantum } \\
\text { de Medição de } \\
\text { Desempenho }\end{array}$ & $\begin{array}{r}\text { Hror } \\
199\end{array}$ & $\begin{array}{l}\text { Avaliar o "valor" para o cliente, que é o relacionamento entre custo e qualidade, } \\
\text { e o "serviço" para o cliente, que é o relacionamento entre qualidade e tempo. As } \\
\text { medidas de desempenho são vinculadas à estratégia e abrangem três níveis (orga- } \\
\text { nizacional, processos e pessoas); há ênfase na satisfação dos principais grupos de } \\
\text { interessados (clientes, acionistas, funcionários, fornecedores, órgãos controlado- } \\
\text { res, sociedade e meio ambiente). }\end{array}$ \\
\hline $\begin{array}{l}\text { O Método de } \\
\text { Rummler e } \\
\text { Branche }\end{array}$ & $\begin{array}{l}\text { Rummler e } \\
\text { Branche } \\
1994\end{array}$ & $\begin{array}{l}\text { sempenho e os níveis de de- } \\
\text { mensões críticas (qualidade, } \\
\text { de mensuração; e contempla } \\
\text { o e do trabalho). }\end{array}$ \\
\hline $\begin{array}{l}\text { Skandia } \\
\text { Navigator }\end{array}$ & $\begin{array}{l}\text { L. Edvinsson } \\
1997\end{array}$ & $\begin{array}{l}\text { Fornece uma imagem equilibrada do capital financeiro e intelectual para, a partir dis- } \\
\text { so, criar um valor sustentável, agindo de acordo com uma visão empresarial e com } \\
\text { a estratégia que dela resulta. Identifica os resultados decorrentes de uma estratégia } \\
\text { baseada na criação de conhecimento; trabalha com passado, presente e futuro; e trata } \\
\text { o desenvolvimento do capital intelectual como foco central da concepção do modelo. }\end{array}$ \\
\hline $\begin{array}{c}\text { SIGMA } \\
\text { Sustainability } \\
\text { Scorecard }\end{array}$ & \begin{tabular}{l|} 
tan- \\
titu- \\
tros
\end{tabular} & $\begin{array}{l}\text { Line - Scorecard - visa medir e estabelecer relações sob quatro en- } \\
\text { tabilidade, cliente externo e interno, conhecimento e habilidade. } \\
\text { entabilidade ao invés do aspecto financeiro, bem como prioriza os } \\
\text { m detrimento dos clientes. }\end{array}$ \\
\hline $\begin{array}{l}\text { VCS - } \\
\text { Value Chain } \\
\text { Scoreboard }\end{array}$ & $\begin{array}{l}\text { Baruch Lev. } \\
\text { Philip Bardes, } \\
2001\end{array}$ & $\begin{array}{l}\text { O scoreboard usa uma cadeia de valor, que consiste em três fases: descoberta de } \\
\text { novos produtos ou serviços ou processos, estabilização de exequibilidade tecno- } \\
\text { lógica e comercialização de novos produtos e serviços. Baseia-se, para isso, em } \\
\text { minuciosa pesquisa científica sobre o relacionamento entre intangiveis e valor de } \\
\text { mercado da companhia e em pesquisa sobre as necessidades de informação para } \\
\text { analistas e outros acionistas, sendo simples e abrangente. }\end{array}$ \\
\hline $\begin{array}{c}\text { PP - } \\
\text { Performance } \\
\text { Prism }\end{array}$ & \begin{tabular}{|l|} 
Neely, Adams \\
e Crowe \\
(2001) e por \\
Kennerley e \\
Neely \\
2002 \\
\end{tabular} & $\begin{array}{l}\text { Utiliza o prisma de desempenho para responder a perguntas relacionadas à satis- } \\
\text { fação dos interessados, às estratégias, aos processos, às capacidades e à contribui- } \\
\text { ção dos interessados. Usa diferentes perspectivas de desempenho; confere ênfase } \\
\text { à satisfação dos diferentes grupos de interessados; estabelece um processo claro } \\
\text { de criação de valor; utiliza uma metodologia que facilita alinhamento estratégico, } \\
\text { assim como entender as relações de causa e efeito. }\end{array}$ \\
\hline $\begin{array}{l}\text { PNQ - Prêmio } \\
\text { Nacional da } \\
\text { Qualidade }\end{array}$ & $\begin{array}{c}\text { FPNQ - Funda- } \\
\text { ção para Prê- } \\
\text { mio Nacional } \\
\text { da Qualidade, } \\
2002\end{array}$ & $\begin{array}{l}\text { Fornece uma visão abrangente de como otimizar o desempenho organizacional. É } \\
\text { mais uma ferramenta de diagnóstico do que um sistema de medição. Utiliza me- } \\
\text { canismos de análise crítica do desempenho global, que facilitam a avaliação orga- } \\
\text { nizacional; estimula a participação dos funcionários no processo de avaliação do } \\
\text { SMDO; e fornece informações que facilitam o aprendizado organizacional. }\end{array}$ \\
\hline
\end{tabular}

Quadro 3 - Evolução dos modelos de avaliação de desempenho

Fonte: Elaborado pelos autores 


\subsection{Avaliação de desempenho x cultura organizacional}

Existem evidências de que há uma relação entre a cultura organizacional e o modo como o desempenho é avaliado dentro de uma organização (SCOTT et al., 2003). Observando divesos autores, Bititci et al. (2006) concluíram que:

a) estilos de cultura e gestão organizacionais têm um impacto sobre a forma como sistemas de medição de desempenho são implementados e utilizados;

b) sistemas de mensuração do desempenho podem afetar os estilos de gestão e, em certo ponto, afetar a cultura organizacional.

Em uma de suas pesquisas, Bourne et al. (2002) levantaram três fatores que diferenciavam empresas que haviam implementado com sucesso medidas de desempenho em relação às que não obtiveram sucesso:

a) objetivo - no início do projeto de implantação das medidas de desempenho, o gerente ou diretor de empresas malsucedidas tende a expressar que a finalidade da implantação de medidas de desempenho é melhorar, justamente, as medidas de desempenho de suas empresas. Os diretores de empresas bem-sucedidas tendem a expressar que a implantação de medidas de desempemho possui como finalidade a melhoria da gestão do negócio;

b) estrutura - todas as empresas de sucesso eram propriedade privada. Todas as empresas malsucedidas faziam parte de grupos de capital aberto de maior porte; c) cultura - todas as empresas bem-sucedidas tiveram culturas que poderiam ser consideradas paternalistas. A cultura das empresas malsucedidas não foi descrita desse modo.

A atribuição de "paternalista" para a cultura das empresas foi feita a partir da observação direta do processo de formulação da estratégia. É possível argumentar que ter uma cultura paternalista pode ser benéfico para a implementação de medidas de desempenho e que o medo das consequências da mensuração dos resultados, se menor, reduz a resistência à implementação de tais medidas. $O$ fato de que as implicações da medida foram levantadas como um fator importante durante as entrevistas apoia fortemente esse argumento (BOURNE et al., 2002).

Então, considerando que a cultura organizacional tem grande influência tanto na organização dos sistemas de mensuração de desempenho quanto no sucesso de sua implantação, é válido estudar como os sistemas de medição do desempenho variam em relação à nação da organização.

\section{METODOLOGIA}

Este trabalho foi uma pesquisa de natureza exploratória. De acordo com Gil (2002), a pesquisa exploratória busca proporcionar maior familiaridade com o problema, com o intuito de torná-lo mais explícito.

O método utilizado foi o estudo multicaso, que, segundo Yin (2006, p. 32), é "uma investigação empírica que investiga um fenômeno comtemporâneo dentro do seu contexto da vida real, especialmente quando os limites entre o fenômeno e o contexto não estão claramente definidos". Para este estudo, foram selecionadas, por conveniência, três empresas multinacionais de origens culturais diferentes: alemã, finlandesa e francesa. Samara e Barros (1997) afirmam que 
uma amostra por conveniência é aquela em que os elementos são selecionados levando-se em conta a facilidade de abordagem.

A coleta de dados foi realiza por meio da aplicação de um questionário baseado na pesquisa de Hofstede realizada entre 1969 e 1973, cujos resultados são amplamente reconhecidos e adotados para estudos e aplicações diversas (HOFSTEDE, 2010). Esse questionário foi aplicado com gestores-chave das organizações, isto é, junto a um representante de cada empresa em nível de gerência ou diretoria, que participa diretamente na gestão de performance da organização.

Todos os respondentes tinham três anos ou mais de atuação na área de gestão de performance organizacional. Apesar de atuarem em outros setores, esta pesquisa tratou apenas da área de energia de cada empresa. Essas organizações possuem estruturas consolidadas de gerenciamento de desempenho e grande expressão no mercado de energia. Atendendo à solicitação de confidencialidade, as organizações participantes da pesquisa não terão seus nomes divulgados. Os resultados desta pesquisa são apresentados na seção seguinte deste trabalho.

\section{DISCUSSÃO DOS RESULTADOS}

\subsection{Mensuração da performance organizacional}

As instituições pesquisadas são indústrias multinacionais, de grande expressão mundial e de origens culturais distintas. Trata-se de indústrias que têm influência relevante no mercado de energia. No Quadro 4, a seguir, são demonstradas algumas das características das organizações em estudo.

\begin{tabular}{|c|c|c|c|}
\hline Empresa & Origem & Quantidade de funcionários & Presença global aproximada \\
\hline Empresa A & Alemanha & 362.000 & 190 países \\
\hline Empresa B & França & 93.000 & 100 países \\
\hline Empresa C & Finlândia & 30.000 & 50 países \\
\hline
\end{tabular}

Quadro 4 - Origem, número de funcionários e presença global

Fonte: Elaborado pelos autores.

Além das características já mencionadas, também foram levantados aspectos relativos à mensuração da performance organizacional dentro de cada uma das empresas. Tais aspectos são demonstrados no Quadro 5, exposto a seguir.

\begin{tabular}{|c|c|c|c|}
\hline Questão & Empresa A & Empresa B & Empresa C \\
\hline $\begin{array}{l}\text { Principais meios de co- } \\
\text { municação e incentivo } \\
\text { para que os colabora- } \\
\text { dores se comprometam } \\
\text { com a missão, a visão, } \\
\text { os valores e os objetivos } \\
\text { da empresa. }\end{array}$ & $\begin{array}{l}\text { Periódicos internos, in- } \\
\text { tranet, informações ge- } \\
\text { renciais on-line, confe- } \\
\text { rências internas, vídeos e } \\
\text { imagens, cartas mensais } \\
\text { do Chief Executive Officer } \\
\text { (CEO) e do Chief financial } \\
\text { officer (CFO) e programas } \\
\text { de incentivo. }\end{array}$ & $\begin{array}{l}\text { Mídia interna, intranet, } \\
\text { internet, reuniões "fa- } \\
\text { ce-to-face", reuniões } \\
\text { informais etc. }\end{array}$ & $\begin{array}{l}\text { Integração de novos fun- } \\
\text { cionários, intranet e co- } \\
\text { municados em quadros } \\
\text { de avisos. }\end{array}$ \\
\hline $\begin{array}{l}\text { Principais métodos de } \\
\text { avaliação de desempe- } \\
\text { nho (financeiro e não } \\
\text { financeiros) utilizados } \\
\text { pela empresa }\end{array}$ & $\begin{array}{l}\text { Relatórios gerenciais e } \\
\text { revisões regulares de seg- } \\
\text { mentos de negócio em ní- } \\
\text { veis funcionais de departa- } \\
\text { mento e em nível regional. }\end{array}$ & $\begin{array}{l}\text { Earnings before inte- } \\
\text { rest and taxes (EBIT), } \\
\text { Earnings before inte- } \\
\text { rest, taxes, deprecia- } \\
\text { tion and amortization } \\
\text { (EBITDA) e BPD (um } \\
\text { tipo de Balanced Sco- } \\
\text { recard). }\end{array}$ & $\begin{array}{l}\text { Acompanhamento de ín- } \\
\text { dices financeiros e de flu- } \\
\text { xo de caixa e preparação } \\
\text { de demonstrações finan- } \\
\text { ceiras intermediárias. }\end{array}$ \\
\hline
\end{tabular}




\begin{tabular}{|c|c|c|c|}
\hline Questão & Empresa A & Empresa B & Empresa C \\
\hline $\begin{array}{l}\text { Principais indicadores } \\
\text { de performance utili- } \\
\text { zados pela empresa ou } \\
\text { unidade de negócio em } \\
\text { que atua }\end{array}$ & $\begin{array}{l}\text { Ordens colocadas, vendas, } \\
\text { índice de pedidos de ven- } \\
\text { da, lucro bruto (\%), múlti- } \\
\text { plos de Pesquisa e Desen- } \\
\text { volvimento (P\&D), Aten- } \\
\text { timento ao planejamento } \\
\text { do Sistema de Gestão } \\
\text { Aambiental (\%),EBIT (\%), } \\
\text { taxa de conversão de cai- } \\
\text { xa, Economic Value Added } \\
\text { (EVA), Return on Capital } \\
\text { Employed (ROCE) eEBITDA. }\end{array}$ & Vendas, EBIT e EBITDA. & $\begin{array}{l}\text { Lucro operacional, nú- } \\
\text { mero de funcionários, va- } \\
\text { lores de pedidos recebi- } \\
\text { dos de clientes, liquidez, } \\
\text { ROCE, giro de estoques, } \\
\text { giro de contas a receber. }\end{array}$ \\
\hline $\begin{array}{l}\text { Reporte da performance } \\
\text { internamente. }\end{array}$ & $\begin{array}{l}\text { Relatórios gerenciais, con- } \\
\text { forme calendário padrão } \\
\text { de reportes. }\end{array}$ & $\begin{array}{l}\text { Reuniões mensais de } \\
\text { revisão de resultados } \\
\text { do negócio. }\end{array}$ & $\begin{array}{l}\text { Mediante demonstrati- } \\
\text { vos financeiros padroni- } \\
\text { zados globalmente e en- } \\
\text { viados para consolidação } \\
\text { na matriz mensalmente. }\end{array}$ \\
\hline $\begin{array}{l}\text { Infomações de perfor- } \\
\text { mance reportadas para } \\
\text { matriz; infomações de } \\
\text { performance reportadas } \\
\text { para a administração local } \\
\text { e frequência de reporte. }\end{array}$ & $\begin{array}{l}\text { Calendário padrão de re- } \\
\text { portes e indicadores de } \\
\text { performance mensais. }\end{array}$ & $\begin{array}{l}\text { Reuniões mensais de } \\
\text { revisão de resultados } \\
\text { do negócio. }\end{array}$ & $\begin{array}{l}\text { As informações são unifor- } \\
\text { mes, tanto para a matriz } \\
\text { como para diretoria local, } \\
\text { substancialmente repre- } \\
\text { sentadas por: balanço, de- } \\
\text { monstração de resultados } \\
\text { e abertura do resultado } \\
\text { por linha de negócio. }\end{array}$ \\
\hline
\end{tabular}

Quadro 5 - Aspectos realtivos à mensurção da performance organizacional

Fonte: Dados da pesquisa

Pode-se observar que todas as três empresas mencionaram comunicar a missão, a visão, os valores e os objetivos da organização, utilizando os mais diversos meios. Porém, nenhuma mencionou um mecanismo formal de incentivo para aumentar o nível de compromentimento dos empregados.

Ao tratar do sistema de mensuração do desempenho e dos indicadores utilizados, foi citado apenas pela empresa B um modelo/sistema de avaliação de desempenho conhecido. A empresa $A$ cita alguns relatórios relativos à avaliação de desempenho, mas a maioria dos indicadores utilizados é de natureza estritamente financeira. Foi possível perceber, também, que a empresa C entende a mensuração de desempenho apenas como a medição dos resultados financeiros.

Os resultados das empresas A e C contradizem a literatura de avaliação de desempenho, em que autores consideram os resultados financeiros uma consequência de bons resultados em outras áreas (ECCLES, 1991; KAPLAN; NORTON, 1992) e afirmam que o uso de indicadores não financeiros também é necessário para a melhoria dos processos na organização (FORTUIN, 1988).

No caso do reporte da performance, não ficou claro, em nenhuma das organizações, se existe um feedback para os funcionários sobre os resultados de seus departamentos. Sabe-se apenas que são realizados reportes aos quais os níveis mais altos da adminstração têm acesso.

\subsection{Cultura organizaiconal $x$ avaliação de desempenho}

Com base nos resultados desta pesquisa junto aos representantes das empresas, apresentam-se, de modo resumido, a relação entre as características observadas para cada dimensão cultural e a forma como cada empresa gerencia seu desempenho.

Hofstede (2010) classificou os países com um score (que varia de zero a cem) para cada característica observada. Nas seções seguintes, é possível observar a comparação desses scores com os dados coletados nas entrevistas. 


\subsubsection{Distância do poder}

O Quadro 6, a seguir, demonstra os índices de distância do poder identificados pelo trabalho de Hofstede (2010) para os países onde as empresas estudadas se originam.

\begin{tabular}{|c|c|}
\hline País & Índice de distância do poder \\
\hline Alemanha & 35 \\
\hline França & 68 \\
\hline Finlândia & 33 \\
\hline
\end{tabular}

Quadro 6 - Índice de distância do poder

Fonte: Adaptado de Hofstede (2010)

A esse respeito, perguntou-se aos respondentes o nível de autonomia que possuíam no gerenciamento do sistema de mensuração de desempenho da organização. Os resultados são apresentados na sequência para cada uma das empresas.

\section{Empresa A - origem alemã}

As modificações ou atualizações na mensuração do desempenho são administradas e delegadas de acordo com o escopo de cada unidade, gerência ou localidade. Todas as decisões estratégicas são tomadas com o envolvimento da matriz, e as decisões relacionadas a operações do dia a dia podem ser tomadas livremente. Existem muitos canais disponíveis a qualquer colaborador que queira fazer críticas ou sugestões relacionadas à gestão de desempenho.

Nesse contexto, pode-se afirmar que a influência na hierarquia (ou distância do poder) é moderada na forma como a empresa $A$ administra seu desempenho e que é consistente com os resultados do trabalho de Hofstede, que encontrou um índice de 35 de distância do poder na Alemanha.

\section{Empresa B - origem francesa}

Na empresa B, as modificações ou atualizações no sistema de avaliação de desempenho são definidas pela alta administração e concentradas principalmente no departamento financeiro. Foi identificado, também, que não há muita clareza em relação a quais decisões devem ser tomadas com o envolvimento da matriz ou não, ainda que esta tenha grande envolvimento com o dia a dia das operações.

A partir desses dados, pode-se dizer que a influência na hierarquia (ou distância do poder) na forma como a empresa $B$ administra seu desempenho é mais elevada e que há aderência entre esses resultados e os do trabalho de Hofstede, que encontrou 68 de índice de distância do poder nessa sociedade.

\section{Empresa C - origem finlandesa}

$\mathrm{Na}$ empresa $\mathrm{C}$, as decisões relativas ao sistema de avaliação de desempenho são tomadas por meio de comitês diretores subordinados à matriz. As decisões tomadas localmente são limitadas através de limites de alçada de valores, número de funcionários etc. Adicionalmente, foi possível notar que não é raro encontrar situações em que os funcionários se sintam receosos de expressar desacordo quanto à administração de desempenho devido à influência constante da matriz na administração local.

Nesse contexto, pode-se afirmar que a influência na hierarquia (ou distância do poder) é alta na empresa $C$, o que contradiz os resultados de Hofstede, que, ao analisar a distância do poder na Finlândia, identificou um índice de 33. 


\subsubsection{Individualismo e coletivismo}

O quadro 7 demonstra os índices de individualismo e coletivismo identificados pelo trabalho de Hofstede (2010) para os países onde as empresas contidas em nossa amostra possuem origem cultural.

\begin{tabular}{|c|c|}
\hline País & Índice de individualismo e coletivismo \\
\hline Alemanha & 67 \\
\hline França & 71 \\
\hline Finlândia & 63 \\
\hline
\end{tabular}

Quadro 7 - Índice de individualismo e coletivismo

Fonte: Adaptado de Hofstede (2010)

Empresa A - origem alemã

O processo implantado de governança oferece liberdade suficiente para os funcionários decidirem como organizar seu trabalho. Em termos gerais, pôde-se observar que a empresa $A$ sinalizou que, em sua forma de gerir seu desempenho, há grande valorização de desafios, qualidade de vida e liberdade dos funcionários para organizar seu trabalho. Assim sendo, o alto índice de individualismo para a Alemanha encontrado no trabalho de Hofstede pode ser claramente observado na gestão de desempenho da empresa A.

\section{Empresa B - origem francesa}

Constatou-se que os processos de gestão de desempenho são muito bem-definidos e são acompanhados pela matriz, mitigando, assim, possibilidades de erros. Além disso, os funcionários possuem liberdade para decidirem como organizar seu trabalho. Foi possível notar que existe uma forte aderência entre o alto índice de individualismo encontrado no trabalho de Hofstede para essa sociedade e a forma como a empresa B gere seu desempenho.

Empresa C - origem finlandesa

$\mathrm{O}$ grau de liberdade que a empresa $\mathrm{C}$ apresenta para que os funcionários organizem seu trabalho é alto, e as exigências de formação e aperfeiçoamento dos funcionários não são tão elevadas, apesar de existirem. Dessa maneira, esses dados indicam um índice de individualismo consistente com o índice de 63 para a Finlândia encontrado por Hofstede.

\subsubsection{Masculinidade e feminilidade}

O Quadro 8 demonstra os índices de masculinidade e feminilidade identificados pelo trabalho de Hofstede (2010) para os países onde as empresas contidas em nossa amostra possuem origem cultural.

\begin{tabular}{|c|c|}
\hline País & Índice de masculinidade e feminilidade \\
\hline Alemanha & 66 \\
\hline França & 43 \\
\hline Finlândia & 26 \\
\hline
\end{tabular}

Quadro 8 - Índice de masculinidade e feminilidade

Fonte: Adaptado de Hofstede (2010) 


\section{Empresa A - origem alemã}

Em relação ao gerenciamento do desempenho da empresa $A$, embora os conflitos sejam resolvidos por meio de negociações, é constante a presença de fortes discussões em que certas decisões são inegociáveis e impostas (por um superior ou pela matriz). A partir disso, pode-se observar um perfil com características mais masculinas que femininas na gestão do desempenho da empresa $A$, sendo coerente com os resultados de Hofstede.

\section{Empresa B - origem francesa}

Na empresa B, os conflitos relacionados ao sistema de mensuração do desempenho são geralmente resolvidos por meio de um sistema democrático de compartilhamento de ideias, em que as decisões são dirigidas pelos níveis da alta gestão, o que significa que a presença de características masculinas na forma de administrar a performance não é tão elevada (estas podem ser entendidas como moderadas), resultado este que é alinhado com os do trabalho de Hofstede.

\section{Empresa C - origem finlandesa}

Os conflitos internos relacionados à gestão de desempenho são geralmente resolvidos com a vitória do mais forte, já que a unidade de negócio que gera melhor resultado possui vantagem nas discussões e acaba sendo beneficiada nessas situações. Assim sendo, tal característica é um forte indício de alto grau de masculinidade, o que contradiz os resultados de Hofstede para a Finlândia.

\subsubsection{Controle da incerteza}

O Quadro 9, exposto a seguir, demonstra os índices de controle da incerteza identificados pelo trabalho de Hofstede (2010) para os países onde as empresas contidas em nossa amostra possuem origem cultural.

\begin{tabular}{|c|c|}
\hline País & Índice de controle da incerteza \\
\hline Alemanha & 65 \\
\hline França & 86 \\
\hline Finlândia & 59 \\
\hline
\end{tabular}

Quadro 9 - Índice de controle da incerteza

Fonte: Adaptado de Hofstede (2010)

\section{Empresa $\mathrm{A}$ - origem alemã}

Na empresa A, verificou-se de forma muito clara que, dentro da estrutura de governança da empresa, há diversos manuais de procedimentos a seguir. As decisões tomadas pela alta administração com relação ao monitoramento de desempenho geralmente são muito previsíveis. Nesse contexto, é possível visualizar que a empresa A possui um perfil de alto controle das incertezas, o que pode ser claramente correlacionado com o alto índice de 65 do trabalho de Hofstede.

\section{Empresa B - origem francesa}

Foi possível observar que a empresa B utiliza diversos manuais de procedimentos para os processos de tomadas de decisão, além de haver uma forte delimitação das responsabilidades e de ser praticamente nula a ocorrência de decisões surpreendentes por parte da administração em relação à gestão de performance, uma vez que todos os procedimentos e planos são claramente comunicados previamente. Diante disso, é possível visualizar que a empresa B possui um perfil de alto controle das incertezas, acahdo que pode ser relacionado com o alto índice de 86 identificado por Hofstede. 


\section{Empresa C - origem finlandesa}

Na empresa C, não foi observado um volume muito grande ou regras rígidas quanto a manuais e procedimentos para os processos de gestão de performance, sendo estes monitorados mais por meio da gestão dos administradores do que a aplicação formal das normas no dia a dia das operações. Tais fatos levam a concluir que a empresa $\mathrm{C}$ apresenta um nível moderado de controle da incerteza quanto à gestão de seu desempenho, o que pode ser correlacionado com o índice de 59 identificado pelo estudo de Hofstede.

\section{CONCLUSÕES}

Esta pesquisa teve como objetivo compreender de que modo os aspectos culturais influenciam a forma com que as empresas do ramo de energia de diferentes origens culturais gerenciam seu desempenho. Pretendeu-se relacionar o modelo de cultura organizacional proposto por Hofstede, considerando-se as quatro dimensões - distância do poder, individualismo, masculinidade e aversão à incerteza - com a avaliação de desempenho nas empresas participantes.

Pôde-se observar que os resultados desta pesquisa foram, na maior parte do tempo, coerentes com os níveis das dimensões do trabalho de Hofstede, indicando que existe uma relação entre a origem cultural da empresa e o modo como a avaliação de desempenho é realizada. Porém, o que se destaca são os resultados contraditórios ao que era esperado para a empresa C, em que os índices para distância do poder e grau de masculinidade são aparentemente muito mais altos do que os encontrados por Hofstede.

Uma possível causa dessa diferença seria as limitações da própria ferramenta, que foi criada tendo como base apenas uma organização e em uma época na qual as fronteiras culturais entre as nações eram muito mais rígidas do que são agora. Atualmente, muitos elementos de outras nações são incorporados pelas sociedades e pelas organizações. Nas visistas às empresas, foi possível observar, ainda, que havia pessoas de diversos países.

Entende-se como a principal contribuição desta pesquisa a evidenciação de diferenças de origem cultural na forma como as empresas avaliam seus desempenhos. Dentre suas limitações, está o impossibilidade de generalização empírica, devido à sua metodologia e à forte dependência que o estudo de caso tem em relação às habilidades do pesquisador (YIN, 2006). Outra limitação está relacionada à impossibilidade de isolar todas as variáveis que possam ter influenciado as diferenças encontradas (YIN, 2006).

Como sugestão para pesquisas futuras, propõe-se realizar uma survey com empresas de um mesmo segmento pelo mundo, estudando, assim, os efeitos da globalização e dos choques culturais na avaliação de desempenho organizacional, conforme ressalta Nelly (1999). 


\section{REFERÊNCIAS}

Andriessen, D.; Tissen, R.; Deprez, F. L., The Knowledge Dividend. London: Financial Times Prentice Hall, 2000.

ARDALAN, K. Globalization and culture: four paradigmatic views. International Journal of Social Economics, v. 36, n. 5, 2009, p. 513-534.

ARIZPE, L.. Culture, Governance and Globalization. Development, v. 48, n.1, 2005, p. 35-39.

AZEVEDO, S. G.; MIRANDA L. C. Indicadores de desempenho gerencial mais utilizados pelos empresários: estudo comparativo Brasil-Portugal. In: XXIV Encontro da ANPAD, 2000, Florianópolis. SC. Anais eletrônicos... Florianópolis: ANPAD, 2000. CD-ROM.

BASKERVILLE, R. F. Hofstede never studied culture. Accounting, Organizations and Society, v. 28, 2003, pp. 1-14.

BITICl, U.; MENDIBIL, K.; NUDURUTATI, S.; TURNER, T.; GARENGO, P. Dynamics of performance measurement and organizational culture. International Journal of Operations and Production Management, v. 26, n. 12, 2006, pp. 1325-1350.

BORTOLUZZI, S. C.; ENSSLIN, S. R.; LYRIO, M. V. L.; ENSSLIN, L. Avaliação de desempenho econômico-financeiro: uma proposta de integração de indicadores contábeis tradicionais por meio da metodologia multicritério de apoio à decisão construtivista (mcda-c). Revista Alcance, v. 18, n. 2, Abr-Jun 201, p. 200-218.

BOURNE, M.; KENNERLEY, M.; FRANCOSANTOS, M. Managing Through Measures: a Study of Impact on Performance. Journal of Manufacturing Technology Management, v. 16, n. 4, 2005, pp. 373-395.

BOURNE, M.; NEELY, A.; PLATTS, K.; MILLS, J. The success and failure of performance measurement initiatives: Perceptions of participating managers. International Journal of Operations \& Production Management, v. 22, n. 11, 2002, pp. 1288-1310.

CASAGRANDE, R. M. Dimensões da cultura organizacional no modelo de Hofstede: estudo em uma empresa brasileira internacionalizada. Dissertação (Mestrado) - Universidade Regional de Blumenau, Blumenau, 2009.

Corrêa, H. L. O estado da arte da avaliação de empresas estatais. 1986. Tese (Doutorado em Administração de empresas). Faculdade de Economia, Administração e Contabilidade da Universidade de São Paulo (FEA USP). Universidade de São Paulo, São Paulo.

CORRÊA, H. L.; HOURNEAUX JR., F.; RUIZ, F. M. $A$ evolução dos métodos de mensuração e avaliação de desempenho das organizações. In: XXIX Encontro da ANPAD, 2005, Brasília, DF. Anais eletrônicos... Brasília: ANPAD, 2005. CDROM.

DRUCKER, P. F. Administração lucrativa. 5 ed. Rio De Janeiro: Zahar, 1964.

ECCLES, R. G. The performance measurement manifesto. Harvard business review, v. 69, n.1, 1991, pp. 131-137.

FAGUNNDES, J. A.; FELIU, V. M. R. Tableau de Bord vs. Balanced Scorecard. Revista de Contabilidade do Mestrado em Ciências Contábeis da UERJ, Rio de Janeiro, v.12, n.1, p.1, jan./abril, 2007.

FOGUEL, F. H. S. Influência da cultura social sobre a qualidade e desempenho empresarial: uma análise do modelo de excelência em gestão, da fundação nacional da qualidade. Revista Administração em Diálogo, v. 14, n. 1, 2010, p. 53-93.

FORTUIN, L. (1988). Performance indicators - Why, where and how? European Journal of Operational Research, v. 34, n.1, 1998, pp. 1-9. 
GIL, A. C. Métodos e técnica de Pesquisa social. 5 ed. São Paulo: Ed. Atlas, 1999.

HOFSTEDE, G. H. Culturas e organizações: compreender a nossa programação mental. Lisboa: Edições Sílabo, 2003.

HOFSTEDE, G. H.; HOFSTEDE, G. J.; MINKOV, $M$. Cultures and organizations: software of the mind: intercultural cooperation for survival. 3 ed. New York: McGraw-Hill, 2010.

HOURNEAUX JR., F. Avaliação de desempenho organizacional: estudo de casos de empresas do setor químico. Dissertação (Mestrado) - Faculdade de Economia, Administração e Contabilidade da Universidade de São Paulo (FEA USP). Universidade de São Paulo, São Paulo, 2005.

Kaplan, R. S.; Norton, D. P. The balanced scorecard - measures that drive performance. Harvard Business Review. Boston, v. 70, n. 1, p. 71-79, January- February, 1992.

Putting the Balanced Scorecard to Work. Harvard Business Review. Boston, v. 71, n. 5, p. 134-147, Setembro-Outubro, 1993.

Using a Balanced Scorecard as a

Strategic Management System. Harvard Business Review. Boston, v. 74, n. 1, p. 75-85, January- February, 1996.

A estratégia em ação. 17 ed. Rio de Janeiro: Campus, 1997.

Organização orientada para a estratégia: como as empresas que adotam - Balanced Scorecard prosperam no novo ambiente de negócios. Rio de Janeiro: Campus, 2000a.

. Having Trouble with your Strategy? Then Map It - Harvard Business Review Septemnber-October, pp. 167-175, 2000 b.

Transforming the balanced scorecard from performance measurement to strategic management: part I. Accounting Horizons 2001
. Mapas estratégicos. 1 ed. Rio de Janeiro: Elsevier, 2004a

"Measuring the Strategic Readiness of Intagible Assets," Harvard Business Review, February, pp. 52-63. 2004b

2006.

Alinhamento. Rio de Janeiro: Campus,

KENNERLEY, M. P.; NEELY, A. D. "A Framework of the Factors Affecting the Evolution of Performance Measurement Systems", International Journal of Operations \& Production Management, Vol. 22, No. 11, pp. 1222-1245. 2002a.

\section{The Performance Prism, The} Scorecard for Measuring and Managing Business Success. FT Prentice-Hall, London. 2002b.

KPMG LLP. Achieving Measurable Performance Improvement in a Changing World: the search for new insights. USA: White Paper, 2001.

LINTON, R. Cultura e Personalidade. São Paulo: Mestre Jou, 1945.

LUGOBONI, L. F. Modelos de Avaliação de Desempenho Organizacional em Instituições de Ensino Superior na grande São Paulo. Dissertação (Mestrado) - Universidade Municipal de São Caetano do Sul, São Caetano do Sul, 2010.

MACHADO, D. D. P. N.; SANTOS, A. F.; PINTO, V. M. Dimensões da cultura organizacional no modelo de Hofstede: Aplicações em uma Organização Militar do Exército Brasileiro e um Órgão Público do Poder Judiciário. São Paulo: XII SEMEAD, 2009.

MOREIRA, L. F. Modelo para determinação do grau de globalização da empresa. Tese (Doutorado) Universidade de São Paulo, São Paulo, 2009.

NEELY, Andy. The performance measurement revolution: why now and what next?. International Journal of Operations \& Production Management, v. 19, n. 2, p. 205228, 1999. 
SOBREIRA NETTO, F. Medição de Desempenho Organizacional: um estudo das vantagens e desvantagens dos principais sistemas sob as óticas teórico-acadêmica e de práticas de mercado. Rio de Janeiro: XXXI EnANPAD, 2007.

PODRUG, N; PAVIČIĆ, J. ; BRATIĆ, V. CrossCultural Comparison of Hofstede's Dimensions and Decision-Making Style within CEE Context. From Transition to Sustainable Development: The Path to European Integration, 2006.

PRASAD, A. Mix, Flux and Flows: The Globalization of Culture and its Implications for Management and Organizations. Journal of Global Business issues, v. 1, n. 2, Jul. 2007.

SAMARA, B. S.; BARROS, J. C. Pesquisa de Marketing. São Paulo: Makron Books, 1997.

SANTOS, M. S. M. Gestão do Desempenho: Relevância da Cultura Organizacional. Dissertação (Mestrado) - Universidade de Coimbra, Coimbra, 2011.

SCOTT, T.; MANNION, R.; MARSHALL, D. H. Does organisational culture influence health care performance? A review of the evidence. Journal of Health Services Research \& Policy, v. 8, n. 2, Apr 2003, pp.105-117.

THE FUTURIST. Assessing global trends for 2025. Edition July-August 2009.

YIN, R. K. Estudo de caso: planejamento e métodos. 3ed. Porto Alegre: Bookman, 2005. 